\title{
HEAT CONDUCTION IN ANISOTROPIC MEDIUM WITH PERFECTLY CONDUCTIVE THREAD-LIKE INCLUSIONS
}

\author{
Heorhiy SULYM*, Nataliia ILCHUK ${ }^{* *}$, laroslav PASTERNAK ${ }^{* *}$ \\ *Bialystok University of Technology, Wiejska Str. 45C, 15-351 Bialystok, Poland \\ "Lutsk National Technical University, Lvivska Str. 75, 43018 Lutsk, Ukraine \\ h.sulym@pb.edu.pl, n.ilchuk@Intu.edu.ua, iaroslav.m.pasternak@gmail.com
}

received 21 May 2019, revised 8 January 2020, accepted 10 January 2020

\begin{abstract}
The paper presents a novel approach for the analysis of steady-state heat conduction of solids containing perfectly conductive thread-like inhomogeneities. Modelling of a thread-like heat conductive inhomogeneity is reduced to determination of density of heat distributed along a spatial curve, which replaces the inclusion. Corresponding boundary integral equations are obtained for anisotropic solids with thread-like inclusions. Non-integral terms are computed in a closed form. It is shown that, nevertheless the singularity of the equation is $1 / r$, it is hypersingular, since the kernel is symmetric. Boundary element approach is adopted for solution of the obtained equations. Numerical example is presented for a rectilinear conductive thread, which verifies derived boundary integral equations.
\end{abstract}

Key words: thread-like inclusion, steady-state heat conduction, anisotropy, boundary integral equation, hypersingular

\section{INTRODUCTION}

Thermomechanical problems for inhomogeneities in structural materials have been widely studied in modern scientific literature, since they are closely related to the analysis of effective properties, strength and fracture of composite materials. Berger et al. (2005) presented an analytic fundamental solution for steady-state heat conduction in functionally graded anisotropic medium. Wang et al. (2005) developed a meshless method for the analysis of steady-state heat conduction in anisotropic and inhomogeneous solids. Vales et al. (2016) presented a homogenisation technique for the estimation of effective thermal properties of composite materials and used it for the analysis of experimental data from thermographic measurements. Khan et al. (2016) presented an analytic approach for determination of effective thermal properties of composites with highly conductive inclusions. Kushch et al. (2017) derived an analytic approach for determination of effective heat conduction properties of composite materials with ellipsoidal inclusions.

In general, thermomechanical problems for inhomogeneities can be divided into three main classes with respect to their geometric shape, which determine the approaches used in their analysis. These are: a) bulky inhomogeneities, in which the dimensions in different directions are comparable; b) thin inhomogeneties (ribbons, shells), in which one dimension is much less than two others and c) thread-like inhomogeneities (wires), in which one dimension is much greater than two others.

The class of bulky inhomogeneities includes globular defects of structural elements or globular filament of composite materials, which is rarely used at present. Such inclusions are mainly studied within the general approaches of thermomechanics. Analytic solutions for anisotropic solids with ellipsoidal inclusions are presented by Kushch et al. (2017). Chao et al. (2009) derived an exact solution for heat conduction in three-phase composite materials with elliptical inhomogeneities. Cepite and Jakovics (2008) presented a finite element analysis of heat conduction in inhomogeneous medium with elliptical pores. Lee et al. (2018) obtained an analytic solution for effective thermal properties for a medium containing spherical inclusions with imperfect interface.

Thin ribbon-like, plate-like and wire-like filaments are widely used in modern composite materials, including nano-composites. It should be mentioned that modern carbon nano-filament, for example, graphene, possesses very high thermal conductivity (Balandin et al., 2010), which should be definitely accounted for in the numerical thermomechanical analysis of carbon nanocomposites. However, the study of thin-walled and thread-like inclusions is complicated due to the low accuracy of general numerical approaches (e.g. finite element and boundary element methods) in modelling of thin shapes. Therefore, thin and threadlike inclusions are studied with special approaches (e.g. see Sulim and Piskozub, 2008), which can reduce the number of degrees of freedom accounting for the $2 \mathrm{D}$ or $1 \mathrm{D}$ geometries of such inhomogeneities.

Thin ribbon-like and shell-like inclusions can be studied with the help of discontinuity (jump) function method, which replaces the inhomogeneity with a surface of field discontinuity (see Pasternak et al., 2019). However, this approach cannot be directly applied to the study of thread-like inclusions. To the best of authors' knowledge, there are no publications which provide some special approaches for thermomechanical analysis of thread-like inhomogeneities. There are only some publications which provide analytic and experimental study of nano-wires based on the approaches of theoretical physics (Anufriev and Nomura, 2019; Im et al., 2013).

Therefore, this study provides a straightforward boundary integral equation approach and the boundary element numerical solution strategy for the analysis of steady-state heat conduction in anisotropic medium containing a perfectly conductive threadlike inclusion. It is the first step in the thermomechanical analysis of materials containing thread-like inhomogeneities. 


\section{BOUNDARY INTEGRAL EQUATIONS OF HEAT CONDUCTION OF ANISOTROPIC MEDIUM CONTAINING A THREAD-LIKE INCLUSION}

Consider an anisotropic medium containing a perfectly conductive thread-like inclusion of constant radius $\rho$, in which the median line $L$ is a smooth curve (see Fig. 1).

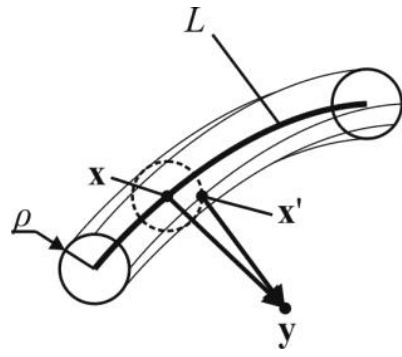

Fig. 1. Thread-like inclusion

Green's third identity for anisotropic heat conduction in a solid with such inclusion is written as (Pasternak et al., 2017)

$$
\begin{gathered}
\theta(\mathbf{y})=\iint_{S}\left(\Theta\left(\mathbf{x}^{\prime}, \mathbf{y}\right) h_{\mathrm{n}}\left(\mathbf{x}^{\prime}\right)-H\left(\mathbf{x}^{\prime}, \mathbf{y}\right) \theta\left(\mathbf{x}^{\prime}\right)\right) \mathrm{d} S\left(\mathbf{x}^{\prime}\right) \\
+\theta^{\infty}(\mathbf{y}),
\end{gathered}
$$

where $\theta$ is temperature, $h_{\mathrm{n}}$ is a heat flux normal to the surface $S$ of the inclusion and $\theta^{\infty}(\mathbf{y})$ is a temperature field due to thermal loading at infinity. Kernels of integral Eq. (1) are defined as (Pasternak et al., 2017)

$\Theta(\mathbf{x}, \mathbf{y})=-\frac{1}{8 \pi^{2}|\mathbf{x}-\mathbf{y}|} \oint_{|\lambda|=1}\left(k_{i j} \lambda_{i} \lambda_{j}\right)^{-1} \mathrm{~d} l(\boldsymbol{\lambda})$,

$H(\mathbf{x}, \mathbf{y})=-k_{i j} n_{i}(\mathbf{x}) \Theta_{, j}(\mathbf{x}, \mathbf{y})$,

where $k_{i j}$ are heat conduction coefficients, $n_{i}(\mathbf{x})$ is a unit normal to the inclusion surface at the point $\mathbf{x}$ and $\boldsymbol{\lambda}$ is a unit vector normal to the position vector $\mathbf{x}-\mathbf{y}$.

When $\rho$ is small compared to a characteristic length $L$ of the thread, one can assume that according to Eq. (2)

$\Theta\left(\mathbf{x}^{\prime}, \mathbf{y}\right) \approx \Theta(\mathbf{x}, \mathbf{y})$

and according to Eq. (3)

$\int_{0}^{2 \pi} H\left(\mathbf{x}^{\prime}, \mathbf{y}\right) \mathrm{d} \vartheta\left(\mathbf{x}^{\prime}\right)=0$,

where $\vartheta$ is the polar angle in the normal cross-section of the inclusion at the point $\mathbf{x}$.

Since the inclusion is assumed perfectly heat conducting, its temperature $\theta(\mathbf{x})$ is a constant, and thus, for small $\rho$ accounting for Eqs (4) and (5), one can rewrite Eq. (1) as

$\theta(\mathbf{y})=\int_{L} \Theta(\mathbf{x}, \mathbf{y}) \gamma(\mathbf{x}) \mathrm{d} L(\mathbf{x})+\theta^{\infty}(\mathbf{y})$,

where

$\gamma(\mathbf{x})=\rho \int_{0}^{2 \pi} h_{\mathrm{n}}\left(\mathbf{x}^{\prime}\right) \mathrm{d} \vartheta\left(\mathbf{x}^{\prime}\right)$

is the sought heat distributed along the inclusion line.

The unknown function $\gamma(\mathbf{x})$ can be determined by solving the integral equation obtained from Eq. (6) by approaching an internal point $\mathbf{y}$ of a medium to some point $\mathbf{x}_{0}$ of the thread-like inclusion:

$\theta^{0}=\lim _{\mathrm{y} \rightarrow \mathbf{x}_{0}} \int_{L} \Theta(\mathbf{x}, \mathbf{y}) \gamma(\mathbf{x}) \mathrm{d} L(\mathbf{x})+\theta^{\infty}\left(\mathbf{x}_{0}\right)$,

where $\theta^{0}$ is the constant temperature of the inclusion, since it is assumed perfectly conductive.

Eq. (8) should be accompanied with the inclusion balance equation

$\int_{L} \gamma(\mathbf{x}) \mathrm{d} L(\mathbf{x})+H_{0}=0$,

where $H_{0}$ is the external heat applied to the inclusion.

It should be noted that, nevertheless the kernel $\Theta(\mathbf{x}, \mathbf{y})$ has singularity $1 / r$, the integral Eq. (8) is hypersingular and cannot be considered as a Cauchy type, since the kernel is symmetric, that is, $\Theta(\mathbf{x}, \mathbf{y})=\Theta(\mathbf{y}, \mathbf{x})$. Therefore, consider this equation in detail.

Assume that the line $L$, which models a thread, is a smooth spatial curve. Introducing an opened contour $L_{\varepsilon}$ of small radius $\varepsilon \rightarrow+0$ surrounding the collocation point $\mathbf{x}_{0}$ (see Fig. 2), Eq. (8) is rewritten as

$\int_{L \backslash L_{\varepsilon}} \Theta\left(\mathbf{x}, \mathbf{x}_{0}\right) \gamma(\mathbf{x}) \mathrm{d} L(\mathbf{x})+B\left(\mathbf{x}_{0}\right) \gamma\left(\mathbf{x}_{0}\right)=\theta^{0}-\theta^{\infty}\left(\mathbf{x}_{0}\right)$,

where

$B\left(\mathbf{x}_{0}\right)=\int_{L_{\varepsilon}} \Theta\left(\mathbf{x}, \mathbf{x}_{0}\right) \mathrm{d} L(\mathbf{x})$.

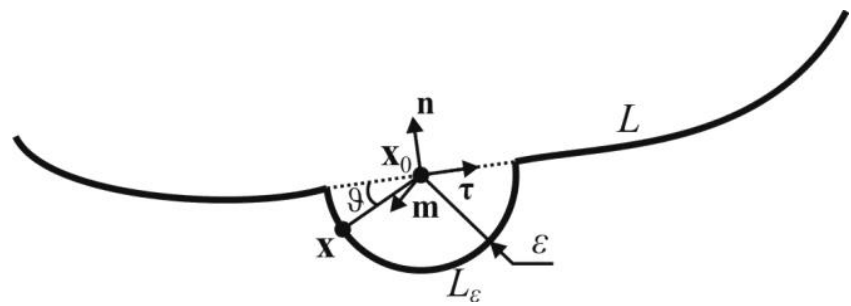

Fig. 2. Determination of the non-integral terms

Substituting Eq. (2) into Eq. (11), one obtains

$B\left(\mathbf{x}_{0}\right)=-\frac{1}{8 \pi^{2}} \int_{0}^{\pi} \oint_{|\lambda|=1}\left(k_{i j} \lambda_{i} \lambda_{j}\right)^{-1} \mathrm{~d} l(\boldsymbol{\lambda}) \mathrm{d} \vartheta$,

where $\lambda(\psi) \perp-(\cos \vartheta \tau+\sin \vartheta \mathbf{n})$. Since according to Eq. (2) the integrand of Eq. (12) is a $\pi$-periodic function of $\vartheta$ and $\psi$, the non-integral term $B\left(\mathbf{x}_{0}\right)$ depends only on the tangent vector $\boldsymbol{\tau}$ to the line $L$ at the point $\mathbf{x}_{0}$.

For isotropic materials, Eq. (12) reduces to

$B\left(\mathbf{x}_{0}\right)=-\frac{1}{4 k}$

and is independent of the spatial orientation of the inclusion line. Here, $k$ is a heat conduction coefficient of an isotropic material.

It should be emphasised that, nevertheless the strength of singularity in Eq. (10) according to Eq. (2) is $1 / r$, the integral in Eq. (10) cannot be computed as the Cauchy principal value. Cauchy principal value can be computed for an integral of a type

$\operatorname{CPV} \int_{a}^{b} \frac{f(x)}{x-x_{0}} d x=$

$\int_{a}^{x_{0}-\varepsilon} \frac{f(x)}{x-x_{0}} d x+\int_{x_{0}+\varepsilon}^{b} \frac{f(x)}{x-x_{0}} d x \quad\left(a<x_{0}<b\right)$, 
but Eq. (10) contains integral of the type

$\int_{\mathrm{a}}^{\mathrm{x}_{0}-\varepsilon} \frac{\mathrm{f}(\mathrm{x})}{\left|\mathrm{x}-\mathrm{x}_{0}\right|} \mathrm{dx}+\int_{\mathrm{x}_{0}+\varepsilon}^{\mathrm{b}} \frac{\mathrm{f}(\mathrm{x})}{\left|\mathrm{x}-\mathrm{x}_{0}\right|} \mathrm{dx} \quad\left(\mathrm{a}<\mathrm{x}_{0}<b\right)$,

which diverges when $\varepsilon$ tends to zero. Therefore, boundary integral equation (11) is hypersingular, and for its solution, the integral in it should be computed as the Hadamard Finite Part.

\section{COMPARISON WITH THE 2D SOLUTION FOR A HEAT CONDUCTIVE LINE INCLUSION}

The studies on 2D heat conductive line inclusions are widely covered in scientific literature (e.g. see Sulim and Piskozub, 2008). However, in $2 D$ heat conduction, line inhomogeneity is not exactly a line in 3D representation. Since a cylindrical (plane heat conduction and plane strain) or a plate-like (generalised heat conduction in a plate with thermally insulated surfaces, plane stress) solid is considered in 2D heat conduction and thermoelasticity, for which the geometry and loading do not change along the generatrix (generally $\mathrm{Ox}_{3}$ axis), a $2 \mathrm{D}$ rigid line is, in general, a cylindrical surface, for which intersection (a $2 \mathrm{D}$ line) with a front plane is considered.

Boundary integral equations for a $2 \mathrm{D}$ heat conducting line are obtained as (e.g. see Sulim and Piskozub, 2008)

$\int_{L} \Theta^{2 \mathrm{D}}\left(\mathbf{x}, \mathbf{x}_{0}\right) \sum \mathrm{h}_{\mathrm{n}}(\mathbf{x}) \mathrm{d} L(\mathbf{x})=\theta^{0}-\theta^{\infty}\left(\mathbf{x}_{0}\right)$,

accompanied with the heat balance equation

$\int_{L} \Sigma \mathrm{h}_{\mathrm{n}}(\mathbf{x}) \mathrm{d} L(\mathbf{x})+H_{0}=0$,

where $\Sigma h_{n}(\mathbf{x})$ is the heat flux discontinuity at transition through the $2 \mathrm{D}$ inclusion line.

The $2 \mathrm{D}$ kernel $\Theta^{2 \mathrm{D}}\left(\mathbf{x}, \mathbf{x}_{0}\right)$ of the integral Eq. (16) possesses logarithmic singularity.

Comparing the boundary integral equations (9) and (10) for a heat conducting thread-like inclusion and Eqs (16) and (17) for a $2 \mathrm{D}$ heat conductive line, one can observe the following.

- Order of singularity. According to Eq. (16), boundary integral equations for $2 \mathrm{D}$ heat conductive line inclusions are weakly singular (with logarithmic singularity). After differentiation of Eq. (16) with respect to $\mathbf{x}_{0}$, one can obtain a singular integral equation with Cauchy principal value integral, which is well studied. In contrast, boundary integral equation (10) for a 3D heat conductive thread is hypersingular with symmetric kernel of the order $1 / r$. The authors have found only one source (Eq. (54) on p. 316 of Polyanin and Manzhirov (2008)), referring the integral equation with a similar kernel.

- Type of equation. It is readily seen that the integral Eq. (10) of a heat conductive thread-like inclusion is a Fredholm equation of the second kind and the integral Eq. (16) of the 2D heat conducting line inclusion is a Fredholm equation of the first kind. Both equations are inhomogeneous.

- Heat flux singularity at inclusion tip. It is well known that the heat flux exhibits the square root singularity at the two tips of the $2 \mathrm{D}$ heat conducting line inclusion (Sulim and Piskozub, 2008). The same concerns the sought solution $\Sigma h_{n}(x)$ of Eq. (16). Heat flux discontinuity $\Sigma \mathrm{h}_{\mathrm{n}}(\mathbf{x})$ at $2 \mathrm{D}$ inclusion line possesses square root singularity at its endpoints. The same concerns the stress field at the $2 \mathrm{D}$ inclusion in thermoelastic problems. Nevertheless, the studies by Mirenkova and Sosnina
(1982) revealed that the stress field near the tips of a rigid needle inclusion possesses another type of singularity. The stresses at the tip of an ellipsoidal needle are of order $O\left(1 /\left(\alpha^{2}|\ln \alpha|\right)\right.$, where $\alpha \ll 1$ defines the curvature of the inclusion at its tips. According to the theory of asymptotic expansions, the same singularity is observed in the stress field in the vicinity of inclusion tips. Also, the same field behaviour is observed in the analysis of flows near thin axisymmetric cavities (see Petrov, 1986).

This comparison reveals that thread-like inclusions should be attributed to a separate class of inhomogeneities, which stands alone from bulky inclusions and shell-like (or 2D line) inclusions. Special mathematical attention should be paid to the development of analytic and numerical approaches of the solution of boundary integral equation (10), which discover and account for the singularity of the sought function at the endpoints of inclusion line.

\section{RECTILINEAR THREAD-LIKE INCLUSION IN ISOTROPIC MEDIUM}

Consider a rectilinear thread-like, heat conductive inclusion of length $2 a$ in an isotropic medium, which is placed at the section $-a<x_{1}<a$ of $O x_{1}$ axis. The heat $h^{0}$ is flowing uniformly along $O x_{1}$ axis. In this case, Eqs (9), (10) and (13) reduce to

$\frac{1}{\pi} \mathrm{HFP} \int_{-a}^{a} \frac{\gamma\left(x_{1}\right)}{\left|x_{1}-x_{1}^{(0)}\right|} \mathrm{d} x_{1}+\gamma\left(x_{1}^{(0)}\right)=-4 h^{0} x_{1}^{(0)}$.

Here, HFP stands for the Hadamard Finite Part of the integral.

Boundary integral equation (18) can be solved numerically with the following boundary element approach. The interval $(-\mathrm{a}, \mathrm{a})$ is divided into $\mathrm{N}$ equal parts (boundary elements). It is assumed that the function $\gamma\left(x_{1}\right)$ is constant on each element $\left(\gamma^{(j)}\right)$. Collocation points $\mathrm{x}_{1}^{(\mathrm{j})}=\mathrm{a}(-1+(2 \mathrm{j}-1) / \mathrm{N})$ are placed at the centre of each boundary element. Thus, the integral equation (18) is reduced to the following system of linear algebraic equations:

$\frac{1}{\pi} \sum_{\mathrm{j}=1}^{\mathrm{N}} \mathrm{a}_{\mathrm{ij}} \gamma^{(\mathrm{j})}=-4 \mathrm{~h}^{0} \mathrm{x}_{1}^{(\mathrm{i})}$,

where

$a_{i j}= \begin{cases}\pi, & i=j \\ \log \left|\frac{t_{i j}+1}{t_{i j}-1}\right|, & i \neq j,\end{cases}$

and $t_{i j}=\left(x_{1}^{(i)}-x_{1}^{(j)}\right)(N / a)$. Here, $i=1, \ldots, N$ and $j=$ $1, \ldots, \mathrm{N}$.

One thousand constant boundary elements of equal length are used to mesh the line of the thread-like inclusion. Numerical results for the sought function $\gamma\left(x_{1}\right)$ are presented in Fig. 3.

Normalised temperature field $\theta k /\left(h^{0} a\right)$ near the tread-like inclusion is presented in Fig. 4 (here, $k$ is the heat conduction coefficient). The field is calculated based on Eq. (2).

One can see in Fig. 4 that the temperature is zero on the inclusion line. Isothermal lines "envelop" the inclusion. Field distribution is very close to the thread. Large field gradient is observed at inclusion tips.

Obtained temperature field was compared with the results of finite element analysis. Perfect agreement was observed, which verifies the proposed approach. 


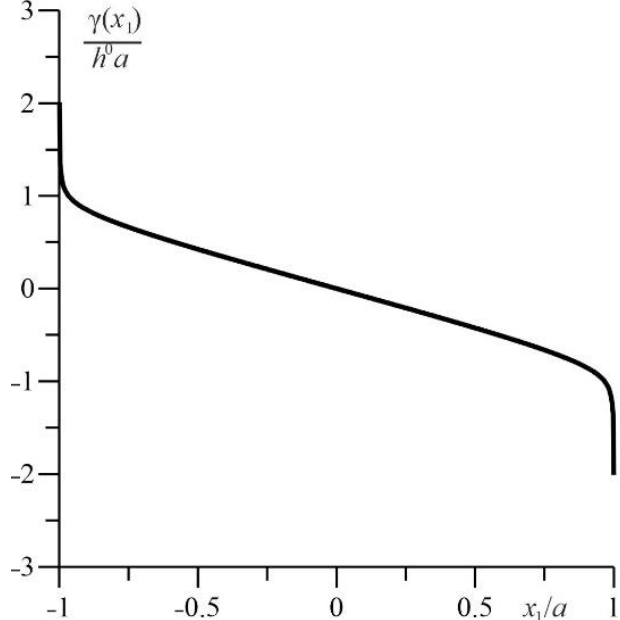

Fig. 3. Distribution of sought heat along the inclusion line

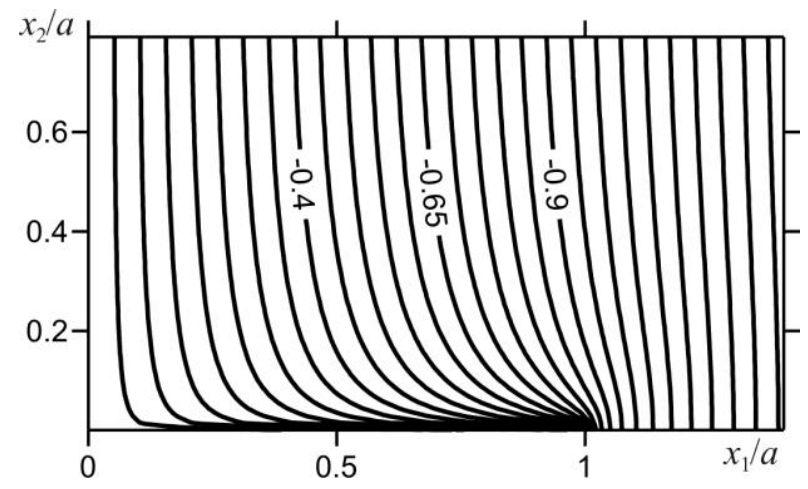

Fig. 4. Dimensionless temperature field near the thread-like inclusion

\section{CONCLUSION}

The study presents a novel approach to the analysis of thread-like inclusions. Boundary integral equations of the problem are obtained and it is shown that, nevertheless the kernel possesses $1 / r$ singularity, this equation is hypersingular.

Numerical solution is presented for a rectilinear thread-like conductive inclusion. Big field gradients are observed near the inclusion line, especially at its tips. Therefore, special studies should be provided on the analysis of singularity of the solution of the obtained hypersingular boundary integral equations.

\section{REFERENCES}

1. Anufriev R., Nomura M. (2019), Coherent thermal conduction in silicon nanowires with periodic wings, Nanomaterials, 9, 142; doi:10.3390/nano9020142.

2. Balandin A.A., Ghosh S., Nika D.L., Pokatilov E.P. (2010), Extraordinary thermal conductivity of graphene: possible applications in thermal management, ECS Trans., 28(5), 63-71.
3. Berger J.R., Martin P.A., Mantič V., Gray L.J. (2005), Fundamental solutions for steady-state heat transfer in an exponentially graded anisotropic material, Z. angew. Math. Phys., 56, 293-303.

4. Cepite D., Jakovics A. (2008), Modelling of a heat tranfer through the material with regular distributed elliptic cavities, HEAT \& POWER AND THERMAL PHYSICS, 1, 56-66.

5. Chao C.K., Chen C.K., Chen F.M. (2009), Analytical exact solutions of heat conduction problemsfor a three-phase elliptical composite, CMES, 47(3), 283-297.

6. Im H., Hwang Y., Moon J.H., Lee S.H., Kim J. (2013), The thermal conductivity of $\mathrm{Al}(\mathrm{OH}) 3$ covered MWCNT/epoxy terminated dimethyl polysiloxane composite based on analytical $\mathrm{Al}(\mathrm{OH}) 3$ covered MWCNT, Composites Part A: Applied Science and Manufacturing, 54, 159-165.

7. Khan K.A., Khan S.Z., Khan M.A. (2016), Effective thermal conductivity of two-phase composites containing highly conductive inclusions, Journal of Reinforced Plastics and Composites, 35, 15861599.

8. Kushch V.I., Sevostianov I., Giraud A. (2017), Local fields and effective conductivity tensor of ellipsoidal particle composite with anisotropic constituents, Proceedings of the Royal Society A: Mathematical, Physical and Engineering Sciences, 473(20170472), 1-19 (http://dx.doi.org/10.1098/rspa.2017.0472).

9. Lee S., Lee J., Ryu B., Ryu S. (2018), A micromechanics-based analytical solution for the effective thermal conductivity of composites with orthotropic matrices and interfacial thermal resistance, SCIENTIFIC REPORTS, 8, Article Number: 7266, DOI: 10.1038/s41598-018-25379-8.

10. Mirenkova G.N., Sosnina E.G. (1982), Rigid ellipsoidal disc and needle in an anisotropic elastic medium, PMM U.S.S.R., 45, 122126.

11. Pasternak la., Pasternak R., Pasternak V., Sulym H. (2017), Boundary element analysis of $3 \mathrm{D}$ cracks in anisotropic thermomagnetoelectroelastic solids, Engineering Analysis with Boundary Elements, 74, 70-78.

12. Pasternak la., Sulym H., Ilchuk N. (2019), Boundary element analysis of 3D shell-like rigid electrically conducting inclusions in anisotropic thermomagnetoelectroelastic solids, Z Angew Math Mech. e201800319 (https://doi.org/10.1002/zamm.201800319)

13. Petrov A.G. (1986), Asymptotic expansions of thin axisymmetric cavities, Journal of Applied Mechanics and Technical Physics, 27(5), 667-672.

14. Polyanin A.D., Manzhirov A.V. (2008) Handbook of integral equations, 2nd ed., Chapman \& Hall/CRC.

15. Sulim G.T., Piskozub J.Z. (2008), Thermoelastic equilibrium of piecewise homogeneous solids with thin inclusions, J Eng Math, $61,315-337$

16. Vales B., Cuartas V.M., Welemane H., Pastor M.-L., Trajin B. (2016), Heat source estimation in anisotropic materials, Composite Structures, 136, 287-296.

17. Wang H., Qin Q.-H., Kang Y.L. (2005), A new meshless method for steady-state heat conductionproblems in anisotropic and inhomogeneous media, Archive of Applied Mechanics, 74, 563-579.

The present paper is financially supported by the Ministry of Science and Higher Education of Poland (research project No S/WM/4/2017) and realised in Bialystok University of Technology. 